\title{
Influence of the pump shape on the modulation instability process induced in a dispersion- oscillating fiber
}

\author{
Christophe Finot ${ }^{1, *}$ and Stefan Wabnitz ${ }^{2}$ \\ 'Laboratoire Interdisciplinaire Carnot de Bourgogne, UMR 6303, 9 av. Alain Savary, 21078 Dijon, France \\ 'Dipartimento di Ingegneria dell'Informazione, Università degli Studi di Brescia, and Istituto Nazionale d'Ottica-CNR, \\ via Branze 38, 25123 Brescia, Italy \\ *Corresponding author: christophe.finot@u-bourgogne.fr
}

Received 19 January 2015; revised 8 March 2015; accepted 9 March 2015; posted 12 March 2015 (Doc. ID 232822); published 27 April 2015

\begin{abstract}
We show that taking into account the temporal intensity profile of a pulsed pump beam may lead to significant deviations from the predictions based on continuous wave analysis of the modulation instability process occurring in a dispersion-oscillating fiber operating under the conditions that lead to gain sideband splitting. Potential application to a pulse doubling scheme with tunable delay is discussed. ( 2015 Optical Society of America
\end{abstract}

OCIS codes: (060.4370) Nonlinear optics, fibers; (190.4380) Nonlinear optics, four-wave mixing; (190.4410) Nonlinear optics, parametric processes.

http://dx.doi.org/10.1364/JOSAB.32.000892

\section{INTRODUCTION}

Modulation instability (MI) is a nonlinear process that has been widely investigated in various fields of physics including plasma, hydrodynamics, and optics, to cite a few. In the presence of a high-power continuous wave (CW), MI leads to the emergence and amplification of gain sidebands in the wave spectrum. In nonlinear fiber optics, such a process has been demonstrated in fibers with anomalous, constant group velocity dispersion (GVD) [1] , as well as in normal GVD fibers by enabling the fulfillment of the nonlinear phase-matching condition through fourth-order dispersion [2], birefringence, or a multimodal structure $[3, \underline{4}]$. More recently, a renewed experimental and theoretical interest in MI studies has been stimulated by the availability of fibers presenting a longitudinal and periodic modulation of their dispersion properties [ 5 ]. Indeed, thanks to the periodic dispersion landscape, which leads to quasi-phase-matching (QPM) of the nonlinear four-wave mixing (FWM) process, MI sidebands can be observed even in the regime of normal average GVD of a dispersion-oscillating optical fiber (DOF) [ $\underline{6}-\underline{8}]$. Recent experiments have confirmed the QPM-induced MI process in the normal GVD regime of microstructure DOF around $1 \mu \mathrm{m}$ [5] , as well as of nonmicrostructure highly nonlinear DOF at telecom wavelengths $[9,10]$. In the case of large-amplitude dispersion oscillations, a process of spectral splitting of the MI spectrum has been highlighted $[\underline{11}, \underline{12}]$, and recently experimentally confirmed [ $\underline{13}]$.

In most of the studies, the theoretical analysis of the first stages of the MI process is based on the assumption of a
$\mathrm{CW}$ with a fixed power $P$. However, from the experimental point of view, in order to reach such a power $P$, it is often required to consider a relatively long (with respect to the MIinduced modulation period) pulsed signal whose peak power is equal to $P[\underline{5}, \underline{2}]$. This is what is often referred as the quasi-CW approximation. It has already been shown that, when using a pulsed pump, the temporal duration of the pump pulses may strongly influence the output signal spectrum: e.g., consider the case of supercontinuum generation [14]. We focus here on the influence of the pump pulse duration in the initial stages of the MI process when no cascading of the process has occurred, and when no coherent temporal structures have emerged. We will consider here three different configurations. The first one corresponds to the standard $\mathrm{MI}$ in a fiber with constant anomalous dispersion. The second configuration deals with the QPM MI that occurs in a DOF with relatively weak dispersion oscillations. Finally, we will investigate the development of $\mathrm{MI}$ in a DOF under the conditions (of strong dispersion oscillations) that lead to the spectral splitting of the first QPM sideband.

As a basis of the discussion, we first recall the results that may be obtained from CW analysis, and we use Floquet linear stability analysis (LSA) in order to predict the instability domains and their evolution according to the peak pump power level. We then discuss the influence of the finite temporal duration of the long pulsed pump. Our conclusions are confirmed by the direct numerical simulation of the nonlinear Schrodinger equation (NLSE). In a last section, we introduce an application that takes advantage of the specific features that 
are experienced by the signal MI gain under the conditions of spectral splitting. Given the power selective nature of the gain process when amplifying a continuous seed, we point out that, within a certain range of parameters, a pulsed pump may lead to the generation of two much shorter signal pulses, whose temporal width and delay can be continuously adjusted by means of controlling the pump power.

\section{ANALYSIS BASED ON A CONTINUOUS WAVE}

\section{A. Model Under Investigation}

The evolution of the optical field in an optical fiber can be described by the NLSE

$$
i \frac{\partial \psi}{\partial z}-\frac{\beta_{2}(z)}{2} \frac{\partial^{2} \psi}{\partial t^{2}}+\gamma|\psi|^{2} \psi=0,
$$

where $\psi$ is the complex electrical field, $z$ is the propagation distance, and $t$ is the reduced time. The NLSE includes both Kerr nonlinearity $\gamma$ and second-order dispersion $\beta_{2}$, which is allowed to periodically oscillate in the longitudinal $z$ direction. In order to illustrate our discussion, let us consider first a CW pump operating at telecommunication wavelengths $(1550 \mathrm{~nm})$, with a power $P$ varying in the range of $0-9 \mathrm{~W}$. The pump is injected in a highly nonlinear fiber (HNLF) with the nonlinear coefficient $\gamma=10 \mathrm{~W}^{-1} \mathrm{~km}^{-1}$.

\section{B. MI Gain Profiles in a Constant Dispersion Fiber}

Let us first consider a HNLF with the constant anomalous dispersion $\beta_{2}=-0.8 \mathrm{ps}^{2} / \mathrm{km}$ and length $L=200 \mathrm{~m}$ (fiber A). The linear gain profile $g_{a n}(\omega)$ of the MI process can be analytically derived from a standard LSA of Eq. (1) as

$$
g_{\text {an }}(\omega)=\left|\beta_{2} \omega\right|\left(\Omega_{C}^{2}-\omega^{2}\right)^{1 / 2}
$$

for $|\omega|<\Omega_{c}$, with $\Omega_{c}$ given by $[\underline{15}]$
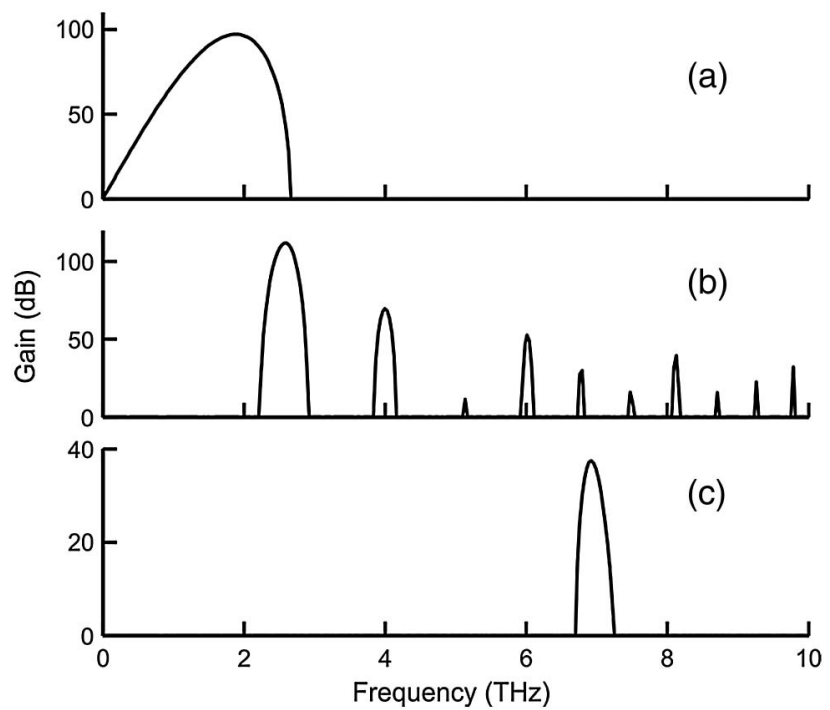

Fig. 1. Evolution of the MI gain profile at the fiber output for a $\mathrm{CW}$ pump power $P=5.6 \mathrm{~W}$ in fibers $\mathrm{A}, \mathrm{B}$, and $\mathrm{C}$ [panels (a), (b), and (c), respectively].

$$
\Omega_{C}^{2}=\frac{4 \gamma P}{\left|\beta_{2}\right|} .
$$

Figure 1(a) illustrates the MI gain profile for a pump power $P=5.6 \mathrm{~W}$. The evolution of the spectrum according to the pump power as predicted by Eq. (2) is plotted in Fig. 2(a). When the pump power grows larger, the frequency of maximum MI gain $\Omega_{m}$ moves toward higher frequencies; moreover, the maximum gain $g_{m}$ grows larger according to the following well-known rules:

$$
\Omega_{m}^{2}=\frac{\Omega_{C}^{2}}{2} \text { and } g_{m}=2 \gamma P .
$$

An important consequence of the previous relations is that the MI gain profile that is induced by a pump of comparatively larger power $P=P_{1}$ fully overlaps the gain profile that is induced by a pump with power $P_{2}$, where $P_{1}>P_{2}$.

\section{MI Gain Profiles in a DOF}

Let us now consider MI in a 400-m-long HNLF with longitudinally oscillating dispersion according to the sinusoidal law

$$
\beta_{2}(z)=\beta_{2 \mathrm{av}}+\beta_{2 \mathrm{amp}} \sin (2 \pi z / \Lambda),
$$

where $\beta_{2 \mathrm{av}}=0.8 \mathrm{ps}^{2} / \mathrm{km}$ is the average fiber dispersion, $\beta_{\text {2amp }}=2 \mathrm{ps}^{2} / \mathrm{km}$ is the amplitude of the dispersion oscillations, and $\Lambda=20 \mathrm{~m}$ is their spatial period (this fiber is called fiber $\mathrm{B})$. The previous parameters are in the same range as the parameters of the HNLF that was experimentally investigated in Refs. $[\underline{10}, \underline{11}]$. The resulting MI leads to the emergence of nonequally spaced sidebands, where the central frequency $\Omega_{p_{-} \text {DOF }}$ of the $p$ th sideband is given by [6]

$$
\Omega_{p_{-} \mathrm{DOF}}^{2}=\frac{2 \pi p / \Lambda-2 \gamma P}{\beta_{2 \mathrm{av}}} .
$$

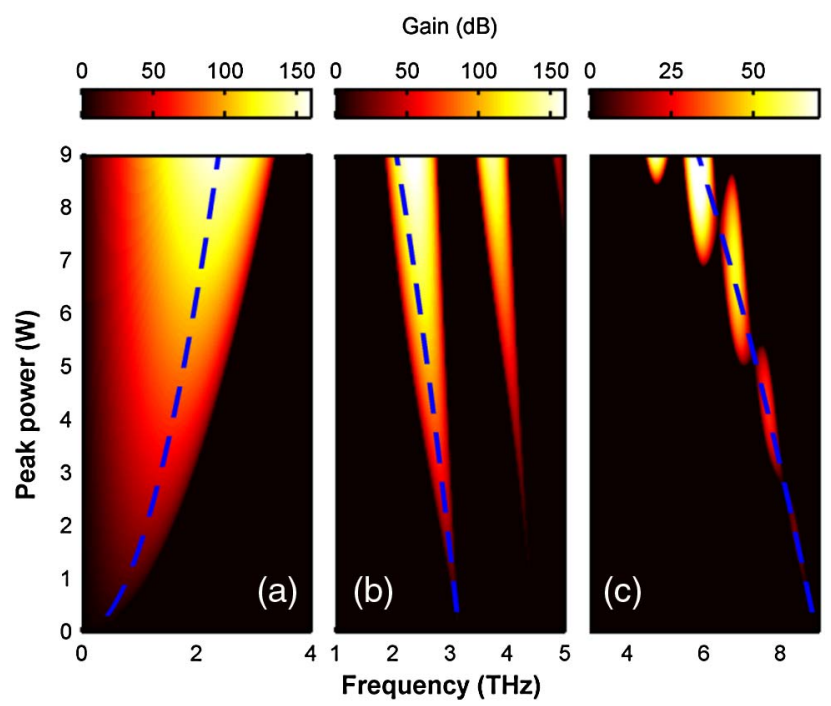

Fig. 2. Evolution of the MI gain profile $g(P, \omega)$ according to pump power $P$ for a CW. (a) Analytical results from Eq. (2) in the case of fiber A. Blue line denotes the maximum MI frequency as predicted by Eq. (4). (b), (c) Results obtained from the LSA using the Floquet approach for fibers B and C, respectively. The resonant frequency predicted by Eq. (6) is denoted by a blue dashed line. 
The MI gain at the resonant frequency $\Omega_{p_{-} \text {DOF }}$ may be estimated in terms of a Bessel function of order $p, J_{p}[\underline{16}]$,

$$
g_{p, \mathrm{DOF}}=2 \gamma P\left|J_{p}\left(\frac{\beta_{2 \mathrm{amp}} \Omega_{p_{-} \mathrm{DOF}}^{2}}{2 \pi / \Lambda}\right)\right| .
$$

Additional insight into the shape of the MI gain sideband may be obtained by taking advantage of a Floquet-based LSA, which has been shown to be a very powerful tool for the analysis of the evolution of the MI gain spectrum in DOFs $[\underline{8}, \underline{11}, 12,17]$. In Fig. 1(b) we plot the output sideband spectrum for a $\mathrm{CW}$ pump power $P=5.6 \mathrm{~W}$. As can be seen, unequally spaced spectral peaks are obtained, with peak values that do not decrease monotonically with the sideband order $p$. A more systematic study of the impact of pump power [Fig. 2(b)] shows that, in agreement with Eq. (6), the central frequencies of the various sidebands decrease as the pump power grows larger. Moreover, in this case there is no longer a complete overlap of the sideband gain profile obtained for a pump power $P$ with the gain profiles that are obtained at pump powers $P^{\prime}<P$.

\section{MI Gain Profiles in a DOF with Sideband Splitting}

By properly adjusting the pump wavelength, it is possible to change the average dispersion of the HNLF, hence controlling the ratio between the dispersion variation amplitude and the average dispersion, or $\beta_{2 \mathrm{amp}} / \beta_{2 \mathrm{av}}$. Let us consider here the regime of strong dispersion oscillations, with $\beta_{2 \mathrm{av}}=0.1 \mathrm{ps}^{2} / \mathrm{km}$ so that $\beta_{2 \mathrm{amp}} / \beta_{2 \mathrm{av}}=20$ (this fiber is called fiber $\mathrm{C}$, and it is of the same length as fiber $\mathrm{B}$ ). In this regime, when $\beta_{2 a m p}$ $(1-\gamma P \Lambda / \pi) / \beta_{2 \mathrm{av}}>3.83$, the standard perturbative analysis resulting in Eq. (7) predicts that the parametric sidebands have a vanishing amplitude. In this context, new, higher-order sidebands, resulting from the splitting of the corresponding original QPM sideband, appear. This phenomenon was first theoretically predicted in [11], and it was recently experimentally confirmed in Ref. [13]. In Fig. 1(c), the $P=5.6 \mathrm{~W}$ pump power is such that a single branch of the first QPM sideband is visible. The results of a more systematic study of the influence of the pump power are reported in Fig. 2(c), which shows that up to five branches of the first QPM sideband can be observed for pump power values between $P=2 \mathrm{~W}$ and $P=5 \mathrm{~W}$. Note that the results of Figs. 1(c) and 2(c), based on the Floquet LSA, will be confirmed in Section 3.C [see Fig. $6(\mathrm{a})]$ by means of the numerical integration of the NLSE.

\section{IMPACT OF PUMPING WITH A FINITE TEMPORAL WIDTH}

\section{A. Situation under Investigation}

Let us investigate now the impact of a quasi-CW pump. We compare different temporal intensity profiles: a Gaussian, a super-Gaussian (of order 3), and a triangular pump pulse. These relatively long pulses (several hundreds of picoseconds in duration) are plotted in Fig. $3(\mathrm{a})$, together with the probability distribution function $\operatorname{pdf}(P)$ of the power that can be associated with those profiles [see Fig. $\underline{3(\mathrm{~b})}$ ]. The peak power of the pulses was chosen to be identical in all cases. The full width at half maximum (FWHM) duration of the Gaussian
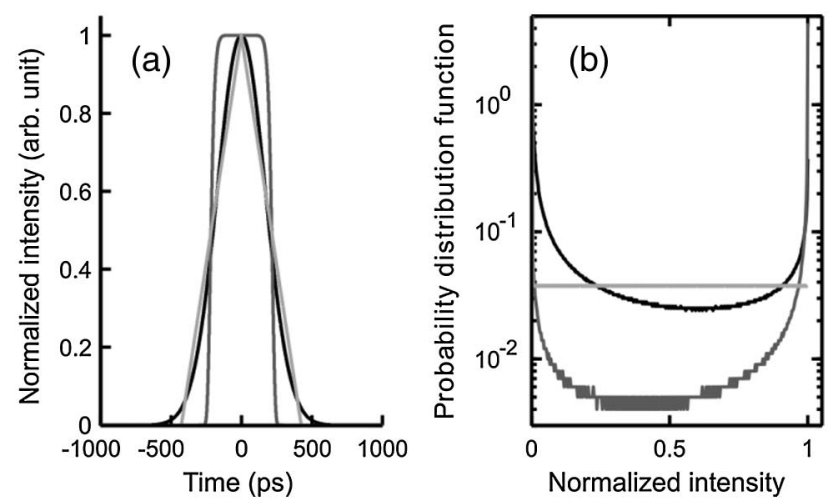

Fig. 3. (a) Comparison of the three temporal intensity profiles of the pulses under investigation. (b) Probability distribution function associated with these profiles (the temporal span of the simulations is 2 ns). Gaussian, super-Gaussian, and triangular shapes are plotted using black solid, gray solid, and light gray solid lines, respectively.

pulse is $400 \mathrm{ps}$, and the temporal width of the other pulses was chosen so that all pulses have the same energy.

\section{B. Qualitative Analysis}

Let us study now the MI spectrum generated by the pulsed pumps of Fig. 3. Given their relatively long duration, we may neglect any dispersion-induced modifications of the temporal intensity of the pump. We may thus consider, as a first approximation, that the pump profile remains constant upon propagation, and that the resulting MI spectrum can be analytically estimated by averaging over the pump power profile; i.e., we use the simple formula

$$
g_{\text {out }}(\omega)=\int g(P, \omega) \operatorname{pdf}(P) \mathrm{d} P .
$$

The resulting spectra that are obtained for a pulsed pump with a peak power of $5.6 \mathrm{~W}$, which is identical to the power value that is used in Fig. 1, are plotted in Fig. 4 .

In the case of the standard MI gain in fiber A [see Fig. 4(a)], we may note that using a pulsed pump has only a moderate influence on the MI gain profile. Indeed, here the shape of the MI spectrum is essentially determined by the pump pulse peak power. Note, however, that the peak gain value may slightly vary with pulse shape, for a constant pump energy. Indeed, whereas the $\mathrm{CW}$ analysis predicts a $90 \mathrm{~dB} \mathrm{MI}$ gain, with a super-Gaussian pump one has nearly $89 \mathrm{~dB}$ of gain. However, the gain drops by as much as $7 \mathrm{~dB}$ with a Gaussian pulse, and by $10 \mathrm{~dB}$ with a triangular pulse.

When considering now the MI gain spectrum that is obtained in a DOF with weak dispersion oscillations [fiber B; see Fig. 4(b)], the influence of the pulse shape also remains quite limited. Except for the previously mentioned MI gain decrease (with respect to the case of a CW pump), the overall MI gain spectrum remains very close to the predictions obtained for a perfectly CW pump, which validates the recent experimental results using nanosecond pump pulses [5,9].

In the case of fiber $\mathrm{C}$, the situation is radically different. Indeed, Fig. 4(c) shows that noticeable differences now appear between the $\overline{\mathrm{MI}}$ gain spectrum that is obtained by the Floquet 

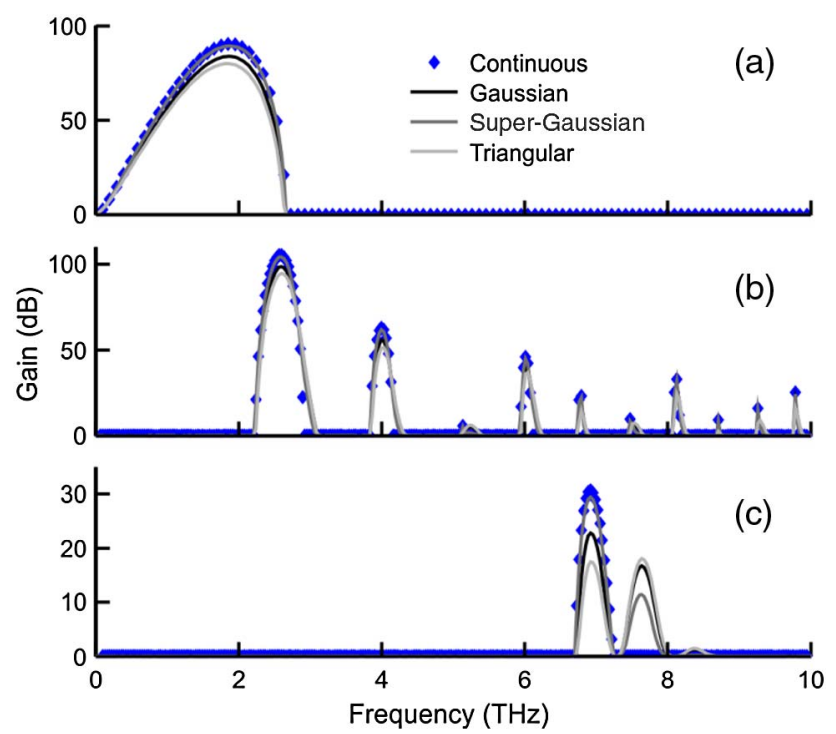

Fig. 4. Details of the output spectra obtained for pulsed pumps having a common peak power of 5.6 W. Results obtained with Gaussian, super-Gaussian, and triangular shapes (black, dark gray, and light gray solid lines, respectively) are compared with results predicted by the LSA for the CW case (blue diamonds). MI spectra obtained in fibers $\mathrm{A}, \mathrm{B}$, and $\mathrm{C}$ are plotted in panels (a), (b), and (c), respectively.

analysis for the CW case and the spectra that are obtained by taking into account the pump shape. Instead of observing a single branch of the first QPM gain sideband, two or three additional gain branches become visible. Whereas for CW pumping, the MI gain vanishes for sideband frequencies higher than the cut-off frequency of $7.3 \mathrm{THz}$, significant gain is still present above this frequency in the secondary branches that are obtained for a pulsed pump. The ratio between the peak values of the inner branch centered at $6.9 \mathrm{THz}$ and the outer branch centered at $7.6 \mathrm{THz}$ crucially depends on the pump pulse shape. For a super-Gaussian pulse, this ratio is of $18 \mathrm{~dB}$, whereas for a Gaussian pulse, the ratio drops down to $6 \mathrm{~dB}$. For a shape having a more peaked central part such as a triangular pulse, the ratio among the inner and outer branches is close to $1(-0.6 \mathrm{~dB})$; i.e., they are of comparable amplitude. Indeed, Fig. 4(c) shows that the value of the inner branch spectral peak gain may change by more than $20 \mathrm{dBs}$ as the pump shape is varied.

To complete our study, we plotted in Fig. $\underline{5}$ the evolution of the sideband gain spectrum as a function of the input peak power of a Gaussian pump as predicted by Eq. ( $\underline{8}$ ). In the case of fibers A and B, the MI gain spectra of Fig. 5 remain qualitatively very similar to their counterparts obtained for a $\mathrm{CW}$ pump as reported in Figs. 2(a) and 2(b). To the contrary, comparing Figs. 2(c) and 5(c) reveals that, in the case of fiber $\mathrm{C}$, the gain spectra for a $\mathrm{CW}$ or a Gaussian pulsed pump are fundamentally different. Indeed, sideband branches that only exist for a finite range of CW pump powers [e.g., consider the branches around 6.9 and $7.6 \mathrm{THz}$, which appear for $\mathrm{CW}$ powers above 5 and $3.2 \mathrm{~W}$, and disappear for $\mathrm{CW}$ powers above 8.7 and $5.8 \mathrm{~W}$ in Fig. 2(c)] no longer vanish at high peak powers in the case of a pulsed pump.

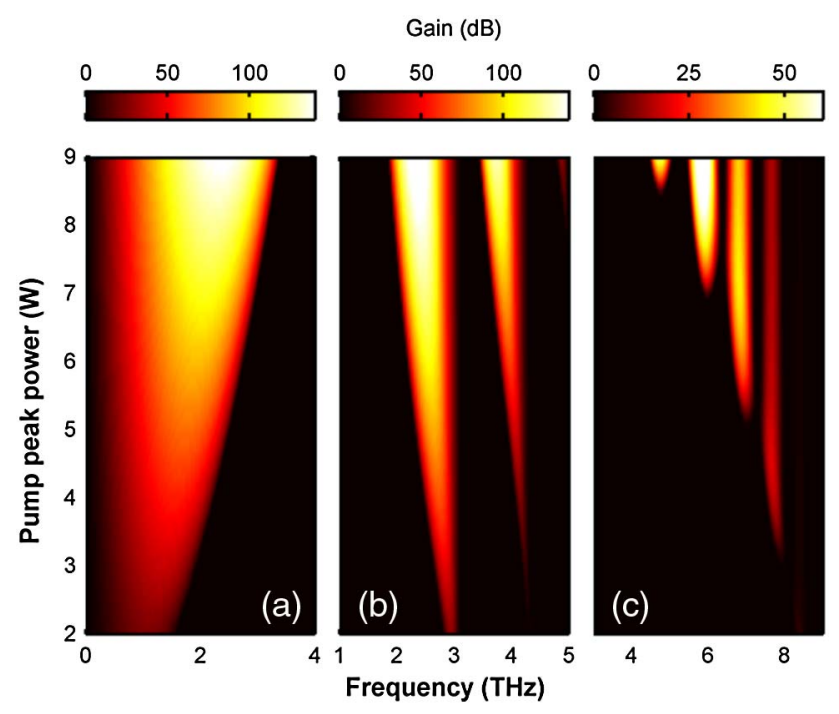

Fig. 5. Evolution of the MI gain profile $g(P, \omega)$ according to the peak power $P$ for a Gaussian pulse in fibers A, B, and C [panels (a), (b), and (c)].

\section{Numerical Simulations}

The previous analysis relies on the approximate approach that is based on the use of Eq. ( 8$)$. However, the pulsed nature of the pump may potentially lead to other nonlinear propagation effects, such as self-phase modulation, which may significantly broaden the pump spectrum. In order to confirm the validity of our conclusions on the MI gain dynamics in fiber $\mathrm{C}$, we numerically solved the NLSE by means of the standard split-step Fourier algorithm, including a weak input white noise seed. Simulations were averaged over 36 shots, and are summarized in Fig. 6 for a CW pump and a Gaussian pump, respectively. The results of the full numerical analysis are in good agreement with the results of the LSA that we discussed in the previous sections. In particular, the persistence of the sideband branches at high pump powers in the case of pulsed pumping is well confirmed.

\section{PULSE DOUBLING AND DELAY TUNING}

\section{A. Pulse Doubling and Intensity Bandpass Filtering}

In Ref. [10] it was experimentally demonstrated that the parametric amplification of a continuous seed in a DOF may be used for the frequency conversion of a nearly sinusoidal pump profile into a train of ultrashort pulses, which are frequency-shifted by several terahertz from the pump. Let us consider now the particular case of using a pump with a finite temporal duration, and a carrier wavelength in the vicinity of the zero dispersion value, so that a spectral splitting of the sideband gain occurs. As we shall see, this situation leads to additional interesting potential applications for the generation of frequency-converted pulse trains. We now add to our simulations a relatively low-power, deterministic $\mathrm{CW}$ seed (e.g., around $0.5 \mu \mathrm{W}$ ) with a frequency offset of $7.6 \mathrm{THz}$ relative to the pump (so that the seed is located in what we previously called the outer branch of the first QPM sideband; 


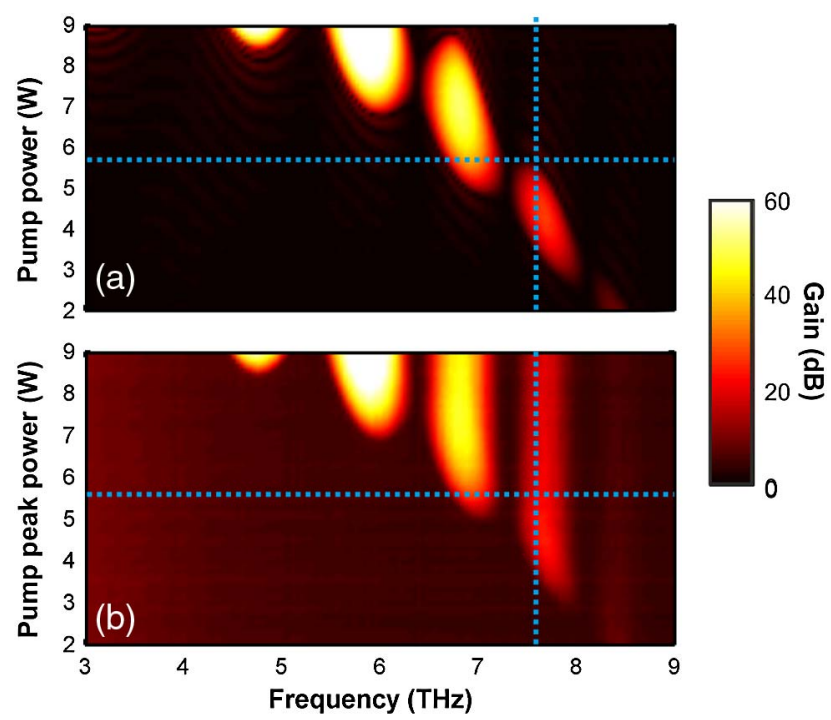

Fig. 6. Evolution of the first QPM band according to pump power for (a) continuous pump and (b) Gaussian pump. Results are obtained from the numerical integration of the NLSE. The horizontal and vertical blue lines indicate the peak power of the pump (5.6 W) and the frequency of the $\mathrm{CW}$ seed $(7.6 \mathrm{THz})$ that are used in Section $\underline{4}$.

see vertical blue line in Fig. 6). When pumped by a $5.6 \mathrm{~W} \mathrm{CW}$, the seed only experiences moderate gain [by solving Eq. (1), we obtain a gain value below $10 \mathrm{~dB}]$. We term here gain or amplification factor the ratio between the peak power of the amplified waveform in the temporal domain and the power of the continuous seed. To the contrary, Fig. 7(a) shows that, when a Gaussian pump is involved (black dashed line), the seed experiences a gain well in excess of $20 \mathrm{~dB}$, which is consistent with Fig. 6(b). Additionally, the most noticeable property of the seed amplification process is that the resulting seed temporal waveform [see the black solid line in Fig. 7(a)] does not reflect the shape of the pump profile. Indeed, instead of a single peaked structure, we observe in Fig. $7(\mathrm{a})$ that two
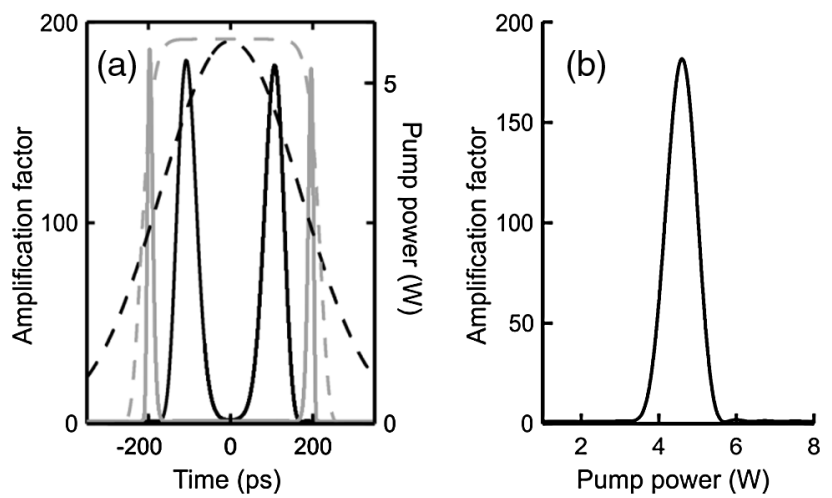

Fig. 7. (a) Time profile of the amplified CW seed when pumped by either a Gaussian pulse (black curve) or a super-Gaussian pulse (gray curve). The temporal profiles of the seed after amplification (solid curves) are compared with the input pump profiles (dashed curves, left). (b) Amplification factor versus instantaneous pump power. well-separated pulses with temporal durations much shorter than the pump ( 55 ps against of 400 ps) are generated. The physical mechanism for the generation of the amplified seed temporal pulse doublet is very different from the mechanisms that lead to the generation of pulse pairs, e.g., in fibers with constant anomalous dispersion $[18,19]$, in SPM or XPM driven processes $[20,21]$, or in the soliton splitting occurring in a DOF with anomalous dispersion [22-24]. Here, as we may clearly see from Fig. $7(\mathrm{~b})$, the double peak structure in the signal pulse is associated with the pump power selectivity of the MI gain process: only a small range of instantaneous power values across the pump pulse profile provides a large amplification of the continuous seed. Such power-dependent filtering behavior leads to the emergence of symmetric peaks both in the leading edge as well as in trailing edge of the signal seed waveform. In contrast, the central portion of the pump pulse (whose power lies above the range of powers that lead to signal gain) has no contribution to the seed amplification. Note that such a bandpass power filtering property (when considering the amplification factor that is associated with each input pump power value) cannot be achieved in the standard MI process that occurs in fibers with constant anomalous dispersion: in that situation, only high-pass nonlinear transfer functions can be implemented.

The spectrum obtained after amplification of the continuous seed by a Gaussian pump is plotted in Fig. 8(a1), with details of the amplified seed given in Fig. 8(a2). We may observe the spectrally broadened seed and idler, as well as the growth of amplified spontaneous emission (ASE) noise,
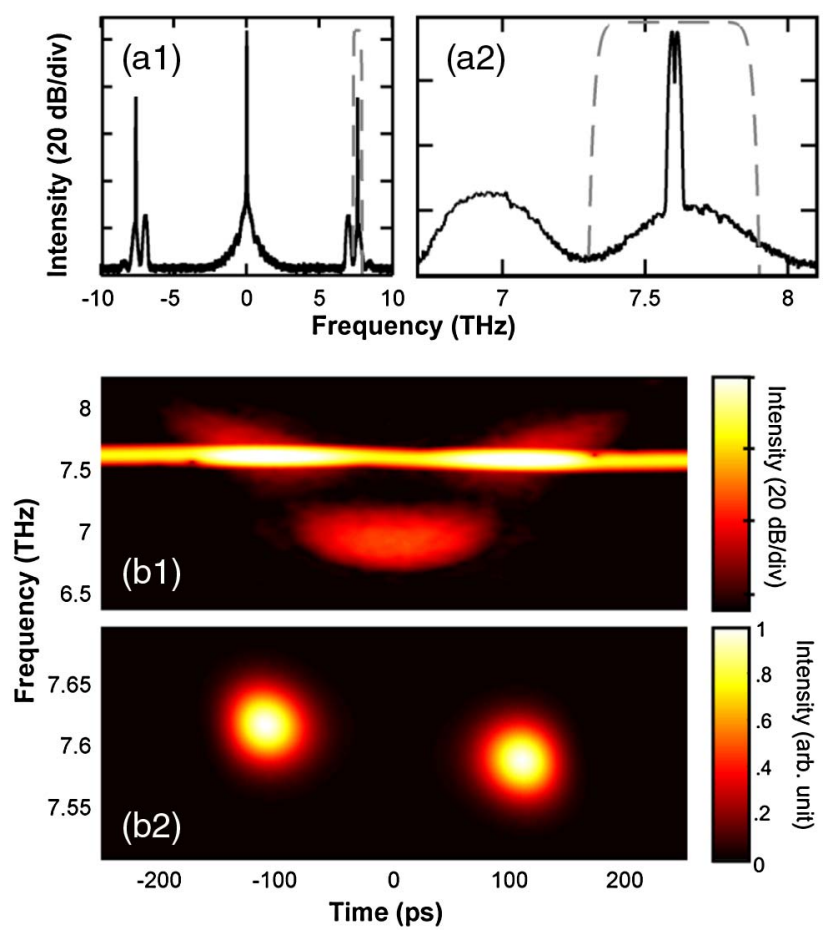

Fig. 8. (a) Optical spectrum of the amplified seed when pumped by a 400 ps Gaussian pulse (black curve) and optical bandpass filter used to isolate the amplified seed (gray line). Plot (a2) is a magnification of plot (a1). (b) Spectrogram of the amplified seed on a logarithmic scale (b1) and its magnification with results on a linear scale (b2). 
mainly in the second QPM sidebands. In order to better understand the spectro-temporal structure of the pulse doublet, we have also computed its spectrogram. From Fig. 8(b1), we note that most of the ASE noise is temporally concentrated in between the two pulses, where the instantaneous power of the pump is close to its maximal value. As a consequence, this ASE that is spectrally located around $7 \mathrm{THz}$ should not dramatically impair the quality of the generated doublet, since it can be easily removed by the bandpass optical filter (OBPF) that is required to isolate the seed or the idler. Magnification of the spectrogram in Fig. 8(b2) reveals that the two subpulses of the amplified seed doublet are not at exactly the same central frequency. The frequency separation of a few tens of gigahertz among the subpulses is explained by the cross-phase modulation that is induced by the leading and trailing edges of the pump pulse [15], and could offer the additional possibility to isolate a single pulse by using a narrow OBPF.

Once again, the details of the intensity profile of the pump pulse are of crucial importance for establishing a bandpass nonlinear power transfer function. When using a super-Gaussian pulse [gray lines, Fig. 7(a)] of the same energy and peak power as the previously considered Gaussian pulse, the FWHM duration of the resulting seed pulses is decreased down to $17 \mathrm{ps}$ (corresponding to a 25 -fold compression compared to the input pump pulse duration), whereas the delay between the two seed pulse components is increased to nearly 400 ps. Such a difference in the temporal width is essentially explained by the increasingly abrupt edges of the super-Gaussian pump when compared with the Gaussian pump pulse.

When considering super-Gaussian pump pulses with a peak power of $6 \mathrm{~W}$, it is interesting to point out that the previously discussed seed pulse doubling process may be efficiently used to convert an initial $1.25 \mathrm{GHz}$ ps pump pulse train into a $12.5 \mathrm{ps}$, $2.5 \mathrm{GHz}$ pulse train with moderate pulse-to-pulse fluctuations (below $5 \%$ of amplitude jitter) and a very high duty cycle ratio (see Fig. 9).

In the additional simulation shown in Fig. 10(a), we show that the pulse doubling effect is not restricted to pump pulses with durations of several hundreds of picoseconds. Indeed, even with a FWHM pump pulse duration of 17.5 ps, which is

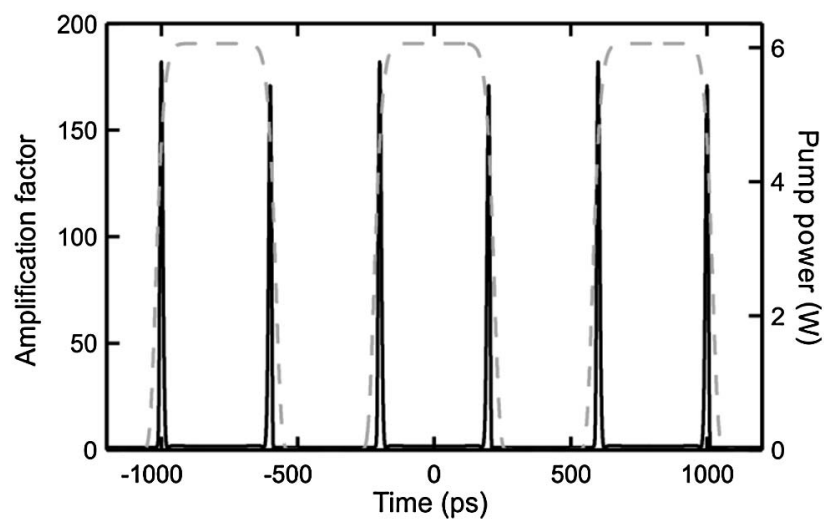

Fig. 9. Amplification factor experienced by the CW seed (solid black curves) compared against the temporal pump power profile (dashed gray curves) for a super-Gaussian pump pulse train similar to Fig. $7(\mathrm{a})$ at the repetition rate of $1.25 \mathrm{GHz}$.
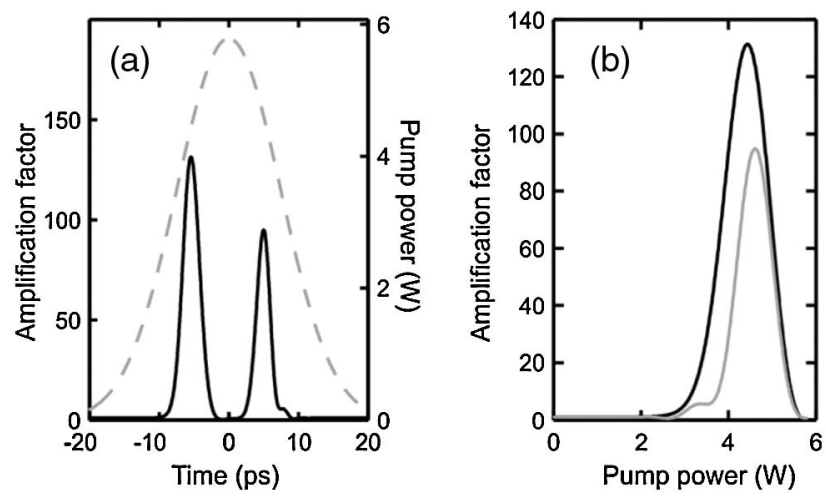

Fig. 10. (a) Amplification factor experienced by the CW seed pulse (solid black curves) compared against the temporal pump power profile (dashed gray curves) for 17.5 ps FWHM duration Gaussian pump pulses. (b) Amplification factor versus instantaneous pump power for signal pulses generated at the leading edge (solid black curve) or the trailing edge (gray curve) of the pump pulse.

typical of $40 \mathrm{GHz}$ communication systems, two 2.5 ps signal pulses are generated. However, as the pump temporal width decreases, the resulting signal temporal structures exhibit an increased temporal asymmetry. Indeed, as clearly outlined by Fig. 10(b), the subpulses that are generated in correspondence with the leading and trailing edges of the pump experience different levels of amplification. Such temporal asymmetry is explained by a combination of a several factors acting simultaneously: temporal walk-off between the pump and the seed, cross-phase modulation induced by the pump on the seed, and spectral broadening of the pump due to self-phase modulation. Moreover, a comparison between Figs. 7(a) and 10(a) shows that the seed amplification factor drops significantly as the pump duration is reduced.

\section{B. Delay Tuning}

By increasing the pump pulse power from 5.3 to $8 \mathrm{~W}$ (Fig. 11), we may observe several additional interesting properties of the seed pulse doubling process. Indeed, the time delay between the two seed pulses can be continuously tuned between 180 and 360 ps by simply adjusting the input power of the 400 ps Gaussian pump [see Figs. 11(a1) and 12(a)]. We should emphasize that the present physical mechanism that introduces a relative time delay of the frequency-converted pulses strongly differs from the usual delay-line schemes that are associated with combining parametric wavelength conversion and dispersion $[\underline{25}, \underline{26}]$ or from the scheme taking advantage of soliton fission in a DOF $[23,24]$. When compared with many fiber-based slow-light devices [27], our approach has the advantage that the tuning of the temporal delay is immune to any significant variations of the peak power of the pulse pair. On the other hand, the temporal width of the generated pulses exhibits a monotonic decrease, from 65 to 32 ps FWHM durations [see Fig. 12(b)]. The optical spectrum of the amplified seed is also influenced by the peak power: as shown in Fig. 11(c), and in agreement with the mechanism of pumpinduced XPM, the spectral splitting previously outlined in Fig. $\underline{8}$ increases as the pump peak power grows larger $[\underline{20}, \underline{21}]$. 

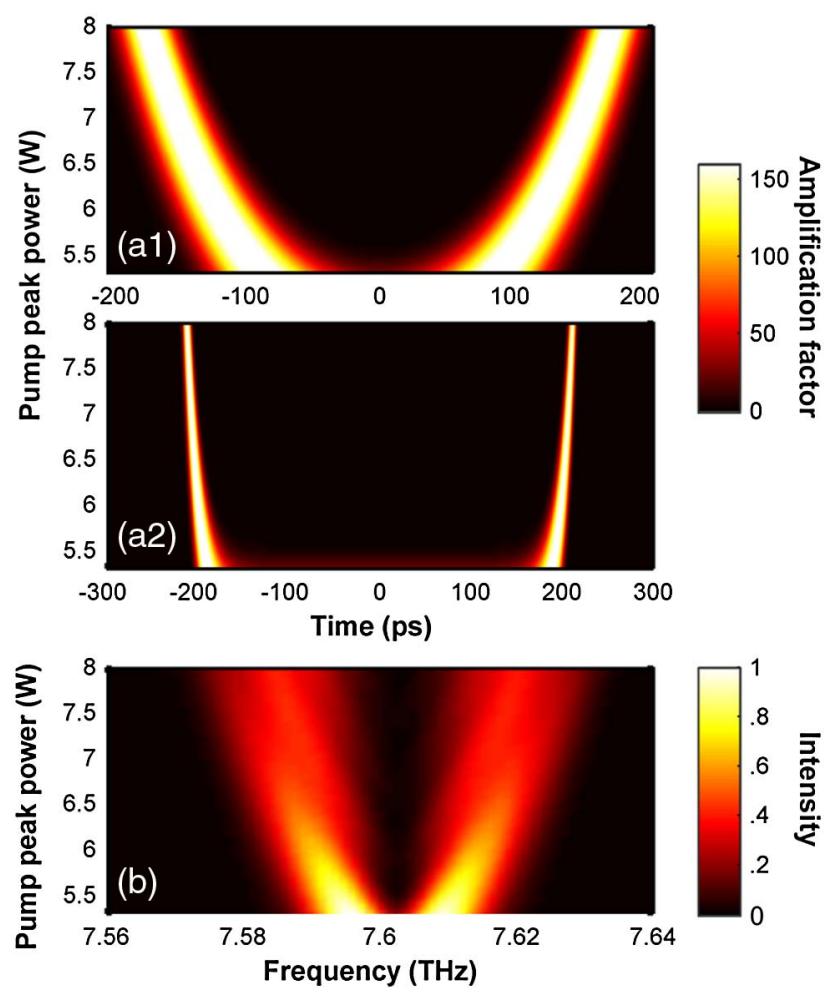

Fig. 11. (a) Evolution of the seed temporal intensity profile versus the pump peak power for (a1) Gaussian and (a2) super-Gaussian pump profiles. (b) Evolution of the seed spectral intensity profile versus the pump peak power for a Gaussian pump profile.

When considering a super-Gaussian pump pulse [Figs. 11(a2) and 12, dashed curves], we may note that relatively longer delays and shorter pulses can be achieved when compared with the Gaussian pump pulse case, but at the cost of a reduced range of tunability. As far as the temporal jitter that is induced by unwanted fluctuations of the peak power of the pulse pump is concerned, we may predict the impact of the pump fluctuations by estimating from Fig. 12(a) the derivative of the delay with respect to the peak power, i.e., the slope of the curves. It then appears that a pulse pair induced
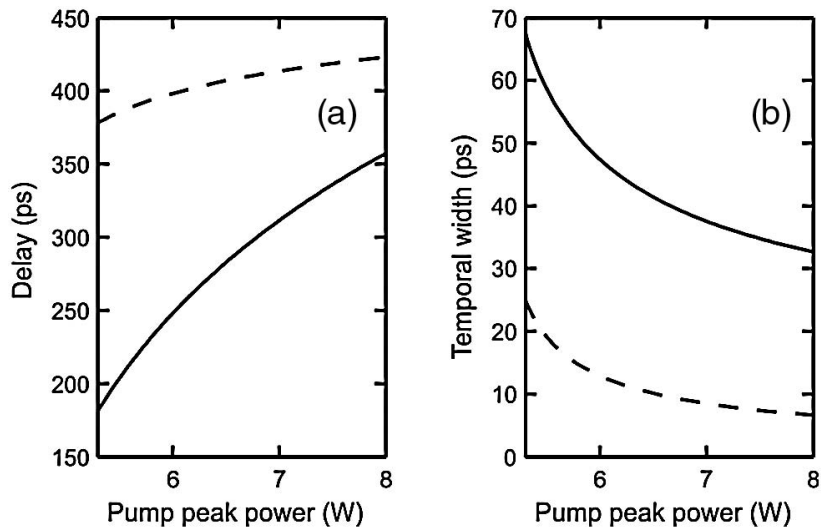

Fig. 12. Evolution of the time delay among the two pulses obtained from the seed (a) and of the FWHM temporal width of the individual pulses (b) versus the pump peak power for either a Gaussian (black curves) or a super-Gaussian (dashed curves) pump pulse shape. by a super-Gaussian pump (dashed curves in Fig. 12) should be relatively less affected by temporal jitter than a pulse pair that is induced by a Gaussian pump (solid curves in Fig. 12).

\section{CONCLUSION}

In this article we have explored the significant influence that the temporal profile of a pulsed pump may have on the MIinduced gain spectrum in specially designed optical fibers with a periodically varying dispersion profile along the longitudinal direction. If the quasi-CW approximation may hold for describing the MI process in fibers with constant dispersion or in DOFs with a weak to moderate amplitude of dispersion oscillations, much more care should be taken when dealing with a pulsed source operating close to the zero dispersion wavelength of a DOF. Indeed, we have shown that in this situation the details of the probability distribution function (pdf) of the pump may strongly affect the spectral splitting of the MI gain spectrum. Moreover, using a pulsed pump beam leads to the conservation of branches that would otherwise disappear under $\mathrm{CW}$ pumping conditions. Combined use of the results of the LSA and of the pdf of the pump could be extended to take into account the statistical properties of a partially coherent pump with a low level of incoherence $[9,28,29]$.

When considering the amplification of a seed signal with a suitable frequency offset from the pump, we have described a novel all-optical signal processing functionality. Namely, the possibility to achieve pulse splitting or doubling, associated with large compression ratio enhancements. The relative time delay between the generated signal pulse doublet is continuously tunable along with the time duration of the individual subpulses, with negligible signal peak power distortion. The continuous adjustment of the delay between the two signal replicas as the pump power is varied may find relevant applications in pump-probe experiments. All of these properties are associated with the use of a DOF with strong dispersion oscillations, operating close to the point of spectral splitting of the MI gain spectrum.

Conseil Regional de Bourgogne (Pari Photcom); French National Research Agency (ANR) (ANR-11-LABX-01-01); Italian Ministry of University and Research (2012BFNWZ2).

We thank Julien Fatome, Kamal Hammani, Yanne K. Chembo, Bertrand Kibler, and Alexej Sysoliatin for stimulating discussions on the subject of dispersion-oscillating fibers.

\section{REFERENCES}

1. K. Tai, A. Hasegawa, and A. Tomita, "Observation of modulational instability in optical fibers," Phys. Rev. Lett. 56, 135-138 (1986).

2. S. Pitois and G. Millot, "Experimental observation of a new modulational instability spectral window induced by fourth-order dispersion in a normally dispersive single-mode optical fiber," Opt. Commun. 226, 415-422 (2003).

3. G. Millot, S. Pitois, P. Tchofo-Dinda, and M. Haelterman, "Observation of modulational instability induced by velocity-matched cross-phase modulation in a normally dispersive bimodal fiber," Opt. Lett. 22, 1686-1688 (1997).

4. G. Millot and S. Wabnitz, "Nonlinear polarization effects in optical fibers: polarization attraction and modulation instability," J. Opt. Soc. Am. B 31, 2754-2768 (2014). 
5. M. Droques, A. Kudlinski, G. Bouwmans, G. Martinelli, and A. Mussot, "Experimental demonstration of modulation instability in an optical fiber with a periodic dispersion landscape," Opt. Lett. 37, 48324834 (2012).

6. N. J. Smith and N. J. Doran, "Modulational instabilities in fibers with periodic dispersion management," Opt. Lett. 21, 570-572 (1996).

7. F. K. Abdullaev, S. A. Darmanyan, A. Kobyakov, and F. Lederer, "Modulational instability in optical fibers with variable dispersion," Phys. Lett. A 220, 213-218 (1996).

8. A. Armaroli and F. Biancalana, "Tunable modulational instability sidebands via parametric resonance in periodically tapered optical fibers," Opt. Express 20, 25096-25110 (2012).

9. C. Finot, J. Fatome, A. Sysoliatin, A. Kosolapov, and S. Wabnitz, "Competing four-wave mixing processes in dispersion oscillating telecom fiber," Opt. Lett. 38, 5361-5364 (2013).

10. F. Feng, J. Fatome, A. Sysoliatin, Y. K. Chembo, S. Wabnitz, and C. Finot, "Wavelength conversion and temporal compression of a pulse train using a dispersion oscillating fibre," Electron. Lett. 50, 768-770 (2014).

11. C. Finot, F. Feng, Y. K. Chembo, and S. Wabnitz, "Gain sideband splitting in dispersion oscillating fibers," Opt. Fiber. Technol. 20, 513-519 (2014)

12. A. Armaroli and F. Biancalana, "Suppression and splitting of modulational instability sidebands in periodically tapered optical fibers because of fourth-order dispersion," Opt. Lett. 39, 4804-4807 (2014).

13. F. Feng, P. Morin, Y. K. Chembo, A. Sysoliatin, S. Wabnitz, and C. Finot, "Experimental demonstration of spectral sideband splitting in strongly dispersion oscillating fibers," Opt. Lett. 40, 455-458 (2015).

14. M. Andreana, A. Labruyère, A. Tonello, S. Wabnitz, P. Leproux, V. Couderc, C. Duterte, A. Cserteg, A. Bertrand, Y. Hernandez, D. Giannone, S. Hilaire, and G. Huss, "Control of near-infrared supercontinuum bandwidth by adjusting pump pulse duration," Opt. Express 20, 10750-10760 (2012).

15. G. P. Agrawal, Nonlinear Fiber Optics, 4th ed. (Academic, 2006).

16. M. Droques, A. Kudlinski, G. Bouwmans, G. Martinelli, and A. Mussot, "Dynamics of the modulation instability spectrum in optical fibers with oscillating dispersion," Phys. Rev. A 87, 013813 (2013).

17. J. C. Bronski and J. N. Kutz, "Modulational stability of plane waves in nonreturn-to-zero communications systems with dispersion management," Opt. Lett. 21, 937-939 (1996).
18. K. Hammani, B. Kibler, C. Finot, P. Morin, J. Fatome, J. M. Dudley, and G. Millot, "Peregrine soliton generation and breakup in standard telecommunications fiber," Opt. Lett. 36, 112-114 (2011).

19. S. Wabnitz and N. Akhmediev, "Efficient modulation frequency doubling by induced modulation instability," Opt. Commun. 283, 1152-1154 (2010).

20. A. Latkin, S. Boscolo, R. S. Bhamber, and S. K. Turitsyn, "Doubling of optical signals using triangular pulses," J. Opt. Soc. Am. B 26, 1492-1496 (2009).

21. N. Verscheure and C. Finot, "Pulse doubling and wavelength conversion through triangular nonlinear pulse reshaping," Electron. Lett. 47, 1194-1196 (2011).

22. A. Sysoliatin, A. K. Senatorov, A. I. Konyukhov, L. A. Melnikov, and V. Stasyuk, "Soliton fission management by dispersion oscillating fiber," Opt. Express 15, 16302-16307 (2007).

23. A. Sysoliatin, A. Belanov, A. I. Konyukhov, L. A. Melnikov, and V. Stasyuk, "Generation of picosecond pulse train with alternate carrier frequencies using dispersion oscillating fiber," IEEE J. Sel. Top. Quantum Electron. 14, 733-738 (2008).

24. R. G. Bauer and L. A. Melnikov, "Multi-soliton fission and quasiperiodicity in a fiber with a periodically modulated core diameter," Opt. Commun. 115, 190-198 (1995).

25. J. E. Sharping, Y. Okawachi, J. van Howe, C. Xu, Y. Wang, A. E. Willner, and A. L. Gaeta, "All-optical, wavelength and bandwidth preserving, pulse delay based on parametric wavelength conversion and dispersion," Opt. Express 13, 7872-7877 (2005).

26. S. Pitois, C. Finot, J. Fatome, and G. Millot, "Design of a continuously tunable delay line using vectorial modulational instability and chromatic dispersion in optical fibers," Opt. Commun. 282, 10161019 (2009).

27. L. Thevenaz, "Slow and fast light in optical fibres," Nat. Photonics $\mathbf{2}$, 474-481 (2008).

28. K. Hammani, C. Finot, and A. Picozzi, "Extreme statistics in Raman fiber amplifier: from analytical description to experiments," Opt. Commun. 284, 2594-2603 (2011).

29. Y. Xu and S. G. Murdoch, "Gain statistics of a fiber optical parametric amplifier with a temporally incoherent pump," Opt. Lett. 35, 626-628 (2010). 